\title{
The ARL of EWMA Chart for Monitoring ZINB Model Using Markov Chain Approach
}

\author{
C. Chananet, S. Sukparungsee, and Y. Areepong
}

\begin{abstract}
In this paper, we proposed the Markov Chain Approach (MCA) to evaluate the average run length (ARL) of exponentially weighted moving average (EWMA) control chart when zero-inflated counted are observed in a negative binomial model. Furthermore, the efficiency of the MCA is compared with Monte Carlo Simulation via the CPU times which the former is much saving computational times used when compared with the latter method.
\end{abstract}

Index Terms-Exponentially weighted moving average, zero-inflated negative binomial, average run length, markov chain, computational times.

\section{INTRODUCTION}

The statistical process control charts are commonly used for monitoring and improving quality of production. The control charts for variables, such as Shewhat $\bar{X}-\mathrm{R}$ control charts, are used as quality characteristic measured on a numerical scale. However, in many quality characteristics cannot be conveniently represented numerically. The quality characteristics of this type are called attributes, for example, in counting the number of defective products or the number of nonconformities in a production process. In this situation, it is necessary to use attribute control charts. Montgomery [1] presented details of explanations and examples for the attribute control charts.

The attribute control charts based on Poisson distribution is used when the observations from a process is a count data. The statistical properties of Poisson distribution have the same mean and variance values and this is not what happens in the real or simulated data. When the variance is greater than the mean, it is so called over-dispersion problem. Thus, the negative binomial distribution can be used instead of Poisson distribution when the data are overdispersed.

Since, the technological advancement in manufacturing processes, more counts of zeros are observed. The excess of zeros under the negative binomial model is called zero-inflated negative binomial (ZINB) model. If we use the c chart for monitoring observation based on ZINB model, the control chart often is underestimated of the values of mean and variance and may lead to higher false alarm rate in detecting out-of-control signal.

The Shewhart control chart has a high performance addition for detecting large shifts, an Exponentially Weighted Moving Average (EWMA) and a Cumulative Sum (CUSUM) control charts were designed to monitor small

Manuscript received May 17, 2014; revised July 17, 2014.

The authors are with the Department of Applied Statistics, King Mongkut's University of Technology North Bangkok, Thailand (e-mail: chanaphunc@kmutnb.ac.th, yupaporna@kmutnb.ac.th). shifts. The CUSUM chart was initially presented by Page [2] and the EWMA chart was introduced by Roberts [3].

There are many literatures available on control charts based on count data. For example, Lucas [4] explained the design and implementation procedure for counted data for detecting of increasing in the count level. Gan [5] proposed a CUSUM chart for binomial counts based on the CUSUM statistics. Borror et al. [6] proposed the EWMA control chart for monitoring Poisson data. Khoo [7] proposed the moving average control chart for monitoring the fraction non-conforming. He et al. [8] presented the CUSUM chart for monitoring a zero-inflated Poisson process. Noorossana et al. [9] applied EWMA chart for monitoring rare health events when some rare health events are zero-inflated binomial distribution.

The Average Run Length (ARL) is a mostly used to measure the performance of control chart. It is the expectation of the time before the control chart gives a false alarm that an in-control process going to out-of-control. The in-control ARL $\left(\mathrm{ARL}_{0}\right)$ should be large for in-control situation, on the other hand, the process need to detect the shift as quickly as possible. Then, the out-of-control ARL $\left(\mathrm{ARL}_{1}\right)$ should be small for out-of-control situation. Many methods for evaluating the ARL for control charts have been studied in many literatures [4]-[6]. A simple method as Monte Carlo simulation is often used to verity and accuracy. Roberts [3] studied the ARL for EWMA chart by using simulations for process base on a normal distribution and derived nomograms that can be used to calculate the ARL for a parameter. Brook and Evans [10] proposed the method to approximate the ARL by using the Markov Chain Approach (MCA) with finite state, and regard intervals of statistics as states of the Markov Chain. Crowder [11] used numerical quadrature methods to solve the exact Integral Equations for the ARL for the ARL for the normal distribution. Sukparungsee [12] have used the Martingale approach to derive an analytical formulas of the ARL when the process are Gaussian and some Non-Gaussian distributions. Areepong [13] derived the explicit formulas of ARL for EWMA control chart in the case of exponential distribution.

In this research, we proposed the closed-form expression of ARL for EWMA chart when observations are from zero-inflated negative binomial model by using the Markov Chain Approach and compared the results obtained from Monte Carlo simulation.

\section{ZERO-INFlated NeGATIVE BINOMIAL MODEL}

The zero-inflated negative binomial model is modeling for count data with combined zero inflation and the negative binomial distribution in inevitable. The appropriate 
probability distribution function to model the condition under study is given in (1). They are the relation of random successful observation with probability $p$ and random number of failure until the successful occur or $X$ which a negative binomial distribution with parameters $\pi, \lambda$ and $r$. We define $X \sim \operatorname{ZINB}(\pi, \lambda, r)$ with the probability mass function (pmf) can be written as

$$
f(x)= \begin{cases}\pi+(1-\pi)\left(\frac{r}{r+\lambda}\right)^{r} & , x=0 \\ (1-\pi) \frac{\Gamma(x+r)}{\Gamma(r) \Gamma(x+1)}\left(\frac{r}{r+\lambda}\right)^{r}\left(\frac{\lambda}{r+\lambda}\right)^{x} & , x=1,2,3, \ldots\end{cases}
$$

where $\pi$ is the probability of zero values, $\lambda$ is the mean of the underlying negative binomial distribution, $\lambda>0$, and $r$ is the over-dispersion parameter where $r \in \mathbb{R}^{+}$. The zero-inflated negative binomial model reduces to zero-inflated Poisson model as $r \rightarrow \infty$.

The mean and variance of the number of failures $(X)$ are given by

$$
E(X)=(1-\pi) \lambda
$$

and

$$
\operatorname{Var}(X)=(1-\pi) \lambda\left(1+\lambda \pi+\frac{\lambda}{r}\right)
$$

\section{EWMA CONTROL CHART FOR ZINB MODEL}

The Exponentially Weighted Moving Average (EWMA) control chart was introduced by Roberts [3]. The EWMA statistics are defined as

$$
Z_{i}=\alpha X_{i}+(1-\alpha) Z_{i-1} \quad i=1,2, \ldots
$$

where $0<\alpha<1$ is a smoothing parameter, $Z_{i}$ is the weighted average between current and previous observations at $i$. The starting value $Z_{0}$ is the process target, so that $Z_{0}=E(X)=\mu_{0}$. If the observations of $X_{i}$ are independent random variables with mean $\mu_{0}$ and variance $\sigma^{2}$, then the variance of $\mathrm{Z}_{i}$ can be shown as follows

$$
\sigma_{Z_{i}}^{2}=\sigma^{2}\left(\frac{\alpha}{2-\alpha}\right)\left(1-(1-\alpha)^{2 i}\right)
$$

The EWMA is constructed by plotting $Z_{i}$ versus the sample number $i$. The upper and lower control limits for the EWMA control chart can be shown as

$$
\mu_{0} \pm A \sigma_{Z_{i}}
$$

where $A$ is the width of control limit.

If the observations are from ZINB model, the expected value and variance of ZINB could be obtained from (6) approaches one as $i$ gets larger. The control limits of ZINB
EWMA control chart can be shown as

$$
\begin{gathered}
L C L=h_{L}=(1-\pi) \lambda-A \sigma \sqrt{\frac{\alpha}{2-\alpha}} \\
U C L=h_{u}=(1-\pi) \lambda+A \sigma \sqrt{\frac{\alpha}{2-\alpha}}
\end{gathered}
$$

where the value $A$ is determined based on a desired in-control $\mathrm{ARL}_{0}$. The ZINB EWMA will signal and out-of-control when $Z_{i}>U C L$ or $Z_{i}<L C L$.

\section{ARL FOR ZINB EWMA CONTROL CHART BY MARKOV CHAIN APPROACH}

The Average Run Length (ARL) is average number of plotted points on a control chart before a point indicates an out-of-control condition [1]. Hence, the ARL is usually used to report the performance of control chart. If the process observations are independent then for any Shewhart control chart, the ARL can be calculated exactly from

$$
A R L=\frac{1}{P(\text { out-of-control signal })} .
$$

Since, the ZINB base on EWMA control chart the plotted points are correlated, the ARL cannot be calculated by (9). The Markov Chain Approach is the effective alternatives to evaluate the ARL which original was proposed by Brook and Evans [10]. Furthermore, it can be applied to ZINB process of EWMA control chart to calculate the ARL. According to this approach, the interval $\left(h_{L}, h_{U}\right)$ in (7) and (8) are divided into $N$ subintervals, so the subinterval's width $(W)$ is $\left(h_{U^{-}} h_{L}\right) / N$. The middle point, $S_{j}$, of the $j^{\text {th }}$ subinterval can be written as

$$
S_{j}=h_{L}+(j-0.5) W
$$

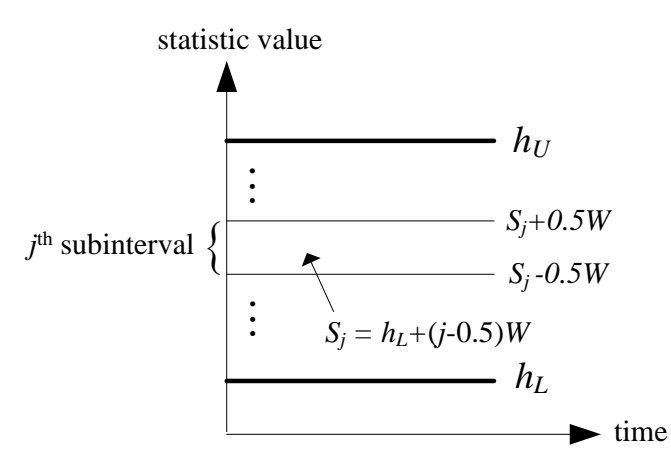

Fig. 1. In-control area is divided into $N$ subintervals.

The statistic $Z_{t}$ in (4), is said to be in transient state $j$ at time $t$ if $S_{j}-0.5 W<Z_{t}<S_{j}+0.5 W$ for $j=1,2, \ldots, N$. If the statistic $Z_{t}$ does not belong to the control limits $\left(h_{L}, h_{U}\right)$, it is said to be in the absorbing state. Hence, the ARL is the expected time to absorption of the Markov chain. The $(N+1)^{\text {th }}$ state represents the absorbing state, which is the out-of-control area below and above the control limits. The one-step transition probability, $P_{i j}$, is the probability at a specific point in time $t, t=1,2, \ldots$ moving from state $i$ at $t-1$ 
to state $j$ at $t$. This transition probability can be written as

$$
\begin{aligned}
P_{i j}= & P\left(S_{j}-0.5 W<Z_{t}<S_{j}+0.5 W \mid Z_{t-1}=S_{i}\right) \\
= & P\left(\frac{1}{\alpha}\left(S_{j}-0.5 W-(1-\alpha) S_{i}\right)<X_{t}<\right. \\
& \left.\quad \frac{1}{\alpha}\left(S_{j}+0.5 W-(1-\alpha) S_{i}\right)\right) \\
= & \Phi_{\text {ZINB }}\left(\frac{1}{\alpha}\left(S_{j}+0.5 W-(1-\alpha) S_{i}\right)\right)- \\
& \Phi_{Z I N B}\left(\frac{1}{\alpha}\left(S_{j}-0.5 W-(1-\alpha) S_{i}\right)\right)
\end{aligned}
$$

where $\Phi_{Z I N B}($.$) is the cumulative distribution function of a$ zero-inflated negative binomial random variable. An exact steady-state probability vector does not exist because the transition probability matrix is not ergodic [14].

Suppose $\mathbf{R}$ contains the probabilities of going from one transient state to another, $\mathbf{I}$ is the $N \times N$ identity matrix, and $\mathbf{1}$ is a column vector of ones. The ARL based on t in-control states is given by (12). Fu et al. [15] proved that,

$$
A R L=\mathbf{p}^{T}(\mathbf{I}-\mathbf{R})^{-1} \mathbf{1}
$$

\begin{tabular}{|c|c|c|c|c|}
\hline$\delta$ & $\begin{array}{c}\mathrm{MCA} \\
\alpha=0.05, \\
\mathrm{UCL}=0.8101\end{array}$ & $\begin{array}{l}\text { CPU time } \\
\text { (seconds) }\end{array}$ & $\begin{array}{c}\text { MC } \\
\text { with standard } \\
\text { deviation }\end{array}$ & $\begin{array}{l}\text { CPU time } \\
\text { (seconds) }\end{array}$ \\
\hline 0.0 & 500.1096 & 21.41 & $502.7897 \pm 0.7202$ & 11229.79 \\
\hline 0.1 & 199.8143 & 21.42 & $200.7955 \pm 0.2785$ & 4698.30 \\
\hline 0.2 & 107.1837 & 21.38 & $108.1993 \pm 0.1444$ & 2687.26 \\
\hline 0.3 & 68.6963 & 21.43 & $68.8023 \pm 0.0888$ & 1847.28 \\
\hline 0.4 & 49.2279 & 21.39 & $48.9604 \pm 0.0615$ & 1429.57 \\
\hline 0.5 & 37.9569 & 21.35 & $37.5461 \pm 0.0461$ & 1192.70 \\
\hline 0.6 & 30.7749 & 21.45 & $30.1946 \pm 0.0364$ & 1041.73 \\
\hline 0.7 & 25.8648 & 21.45 & $25.2435 \pm 0.0301$ & 939.97 \\
\hline 0.8 & 22.3247 & 21.50 & $21.6489 \pm 0.0255$ & 867.54 \\
\hline 0.9 & 19.6649 & 21.35 & $18.9366 \pm 0.0221$ & 811.83 \\
\hline 1.0 & 17.6002 & 21.30 & $16.8270 \pm 0.0196$ & 771.11 \\
\hline 1.5 & 11.7677 & 21.28 & $10.9272 \pm 0.0127$ & 653.73 \\
\hline 2.0 & 9.0645 & 21.34 & $8.1884 \pm 0.0097$ & 595.64 \\
\hline 3.0 & 6.5029 & 21.37 & $5.5796 \pm 0.0069$ & 540.69 \\
\hline 4.0 & 5.2705 & 21.39 & $4.3385 \pm 0.0056$ & 512.04 \\
\hline
\end{tabular}

where $\mathbf{p}^{\mathrm{T}}=(0, \ldots, 0,1,0, \ldots, 0)^{\mathrm{T}}$, is initial state with 1 at the $i^{\text {th }}$ coordinate and zeros elsewhere.

\section{COMPARISON OF PERformance of CONTROL Chart}

TABLE I: COMPARISON OF ARL OF ZINB EWMA WITH MCA AND MC WHEN PARAMETER OF ZINB Is $\pi=0.2, \lambda=0.5$, AND $r=0.5$

To evaluate the performance of control charts, we compare the numerical results for ARL of EWMA control chart in (7) and (8) obtained from Markov Chain Approach (MCA) in (12) and Monte Carlo simulation (MC). We assume that observations are from ZINB model with parameter $\pi=0.2$, $\lambda=0.5$, and $r=0.5$ and 20 . The smoothing constant of EWMA control chart is $\alpha=0.05$. The simulation is based on single sample, repeated 500,000 times for each case by using the $\mathrm{R}$ programming [16] and generate ZINB observations with package VGAM [17]. The performance evaluation of the control chart based on $\mathrm{ARL}_{0}=500$ for the in-control situation and $\mathrm{ARL}_{1}$ for the out-of-control situation, when the process mean shift move from $(1-\pi) \lambda$ to $(1-\pi) \lambda_{1}$, where $\lambda_{1}=\lambda+\delta \sigma, \delta=0.1,0.2, \ldots, 1.0,1.5,2.0,3.0$, and 4.0, respectively. We present the numerical results as shown in Table I and Table II.

In Table I, we will consider the ZINB model with parameter $\pi=0.05, \lambda=0.5$ and $r=0.5$. The numerical results from MCA are in good agreement with the results obtained from MC, however, the MCA uses the CPU times are much less than the MC.

In Table II, the numerical results from MCA are closed to MC and MCA used the CPU time are less than another when the over-dispersion parameter ZINB is increased as $r=20$.

TABLE II: COMPARISON OF ARL OF ZINB EWMA WITH MCA AND MC WHEN PARAMETER OF ZINB IS $\pi=0.2, \lambda=0.5$, AND $r=20$

\begin{tabular}{ccccc}
\hline \hline & MCA & CPU time & $\begin{array}{c}\text { MC } \\
\text { with standard } \\
\text { deviation }\end{array}$ & $\begin{array}{c}\text { CPU time } \\
\text { (second) }\end{array}$ \\
\hline 0.0 & 500.0063 & 1.77 & $499.8654 \pm 0.6983$ & 11237.20 \\
0.1 & 211.5564 & 1.75 & $211.0042 \pm 0.2861$ & 4915.30 \\
0.2 & 110.0596 & 1.71 & $109.1709 \pm 0.1411$ & 2712.65 \\
0.3 & 67.9581 & 1.74 & $67.1224 \pm 0.0819$ & 1808.99 \\
0.4 & 47.1543 & 1.70 & $46.2744 \pm 0.0537$ & 1371.64 \\
0.5 & 35.4104 & 1.73 & $34.4868 \pm 0.0382$ & 1124.10 \\
0.6 & 28.0925 & 1.70 & $27.1213 \pm 0.0288$ & 971.71 \\
0.7 & 23.1819 & 1.73 & $22.2418 \pm 0.0228$ & 872.72 \\
0.8 & 19.6952 & 1.70 & $18.7549 \pm 0.0187$ & 798.91 \\
0.9 & 17.1081 & 1.72 & $16.1226 \pm 0.0156$ & 743.64 \\
1.0 & 15.1206 & 1.71 & $14.1399 \pm 0.0135$ & 702.50 \\
1.5 & 9.6124 & 1.72 & $8.6259 \pm 0.0078$ & 587.47 \\
2.0 & 7.1191 & 1.73 & $6.1357 \pm 0.0055$ & 544.49 \\
3.0 & 4.8007 & 1.71 & $3.8113 \pm 0.0035$ & 485.92 \\
4.0 & 3.7096 & 1.71 & $2.7163 \pm 0.0026$ & 470.25 \\
\hline \hline
\end{tabular}

\section{CONCLUSION}

We proposed the Markov Chain Approach (MCA) for evaluate ARL of EWMA control chart when the observations are ZINB model. The numerical results obtained from MCA are in good agreement the results obtain from Monte Carlo (MC) simulation. Furthermore, the CPU times of MCA is much saving when compared with the MC.

\section{REFERENCES}

[1] D. C. Montgomery, Introduction to Statistical Quality Control, $5^{\text {th }}$ ed., New York, USA : John Wiley, 2005. ch. 6, pp. 264-316.

[2] E. S. Page, "Continuous inspection schemes," Biometrika, vol. 41, pp 100-114, 1954.

[3] S. W. Roberts, "Control chart tests based on geometric moving average," Technometrics, vol. 1, pp. 239-250, 1959.

[4] J. M. Lucus, "Counted data CUSUM's," Technometrics, vol. 27, pp. 29-44, 1985

[5] F. F. Gan, "An Optimal design of CUSUM control charts for binomial counts," Journal of Applied Statistics, vol. 20, no. 4, pp. 445-460, 1993.

[6] C. M. Borror, C. M. Champ, and S. E. Rigdon, "Poisson EWMA control charts," Journal of Quality Technology, vol. 30, no. 4, pp. 352-361, 1993.

[7] M. B. C. Khoo, "A moving average control chart for monitoring the fraction non-conforming," Quality and Reliability Engineering International, vol. 20, pp. 617-635, 2004. 
[8] S. He, W. Huang, and W. H. Woodall, "CUSUM charts for monitoring a zero-inflated Poisson process," Quality and Reliability Engineering International, vol. 28, pp. 181-192, 2012.

[9] R. Noorossana, A. A. Fatahi, P. Dokouhaki, and M. Babakhani, "ZIB-EWMA control chart for monitoring rare health events," Journal of Mechanics in Medicine and Biology, vol. 11, no. 4 pp. 881-895, 2011.

[10] D. Brook and D. A. Evans, "An approach to the probability distribution of CUSUM run length," Biometrika, no. 9, pp. 539-548, 1972.

[11] S. V. Crowder, "A simple method for studying run length distributions of exponentially weighted moving average charts," Technometrics, no. 29, pp. 401-407, 1978.

[12] S. Sukparungsee and A. A. Novikov, "Analytical approximations for detection of a change-point in case of light-tailed distributions," Journal of Quality Measurement and Analysis, no. 4, pp. 373-380, 2008.

[13] Y. Areepong and A. A. Novikov, "Martingale approach to EWMA control chart for changes in Exponential distribution," Journal of Quality Measurement and Analysis, vol. 4, pp. 197-203, 2008.

[14] J. M. Lucus and M. S. Saccucci, "Exponentially weighted moving average control schemes: Properties and enhancements," Technometrics, vol. 32, no. 1, pp. 1-12, 1990.
[15] J. C. Fu, F. A. Spiring, and H. Xie, "On the average run lengths of quality control schemes using a Markov chain approach," Statistics and Probability Letters, vol. 56, pp. 369-380. 2002.

[16] J. C. Fu, F. A. Spiring, and H. Xie, "On the average run lengths of quality control schemes using a Markov chain approach," Statistics and Probability Letters, vol. 56, pp. 369-380, 2002.

[17] R Core Team. (2014). R: A language and environment for statistical computing. R Foundation for Statistical Computing, Vienna, Austria. [Oniline]. Available: http://www.R-project.org/

T. W. Yee. (2012). VGAM: Vector Generalized Linear and Additive Models. $R$ package version 0.9-0. [Oniline]. Available: http://CRAN.R-project.org/package=VGAM.

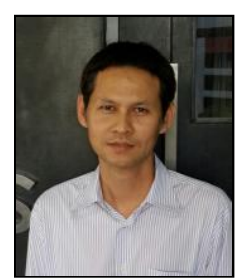

Chanaphun Chananet is currently a Ph.D. candidate in applied statistics, the Department of Applied Statistics, King Mongkut's University of Technology North Bangkok, Thailand. His research interests include statistical process control field and operations research. 